\title{
PSEUDOMONAS SPECIES CAUSING URINARY TRACT INFECTION AND ITS ANTIBIOGRAM AT A TERTIARY CARE HOSPITAL
}

\author{
SHOBHA KL ${ }^{1 *}$, RAMACHANDRA L ${ }^{2}$, AMITA SHOBHA RAO ${ }^{1}$, ANAND KM ${ }^{1}$, GOWRISH RAO S \\ ${ }^{1}$ Department of Microbiology, Melaka Manipal Medical College, Manipal University, Manipal, Karnataka, India. ${ }^{2}$ Department of Surgery, \\ Kasturba Medical College, Manipal University, Manipal, Karnataka, India. Email: shobha.kl@manipal.edu
}

Received: 15 May 2017, Revised and Accepted: 10 July 2017

\section{ABSTRACT}

Objectives: Pseudomonas species cause urinary tract infection (UTI). This study was conducted to isolate Pseudomonas species causing significant bacteriuria and to analyze its antibiogram at a tertiary care hospital.

Methods: About 107 urine samples received in the laboratory from August 2015 to July 2016 having significant bacteriuria with Pseudomonas species were tested for antimicrobial activity. Samples were from both inpatients and patients attending as outpatients to a tertiary care hospital with different age groups and gender. Midstream urine samples were inoculated using a standard calibrated loop into blood agar and MacConkey agar following semi quantitative technique using Kass concept. MALD (Biomerio, ElToile, France) was used for speciation, and Vitex automated system (Biomerio, ElToile, France) was used for antibacterial sensitivity testing.

Results: About 107 urine samples contained Pseudomonas species by MALDI obtained from 69 male (64.48\%) and 38 (35.51\%) female patients. Inpatients were 90 (84.11\%) and 17 (15.88\%) outpatient department (OPD) patients. Isolation of Pseudomonas aeruginosa was from 90 samples $(84.11 \%)$ and Pseudomonas putida in 17 (15.88\%). Significant bacteriuria was more in the age group of $>60$ years in both genders. Susceptibility to gentamicin was 52 (48.59\%), ceftazidime 56 (52.33\%), and imipenem was 58 (54.20\%). Organism was multidrug resistant in 49 (45.79\%) samples.

Conclusion: P. aeruginosa remains the most common uropathogen among Pseudomonas species. Drug of choice for inpatients suffering from UTI by Pseudomonas species can be gentamicin and for patients attending OPD can be ceftazidime. Imipenem can be the reserve drug. Periodic antibiotic review is required for proper treatment of UTI.

Keywords: Pseudomonas species, Urinary tract infection, Semi quantitative method.

(C) 2017 The Authors. Published by Innovare Academic Sciences Pvt Ltd. This is an open access article under the CC BY license (http://creativecommons. org/licenses/by/4. 0/) DOI: http://dx.doi.org/10.22159/ajpcr.2017.v10i11.20002

\section{INTRODUCTION}

Urinary tract infection (UTI) is one of the leading causes of infection worldwide. Although Escherichia coli is predominantly associated with the etiology of UTI [1]. Another organism like Pseudomonas aeruginosa is on the rise [2]. P. aeruginosa has proven to be one of the multidrug resistant organisms thus making its treatment ineffective [3]. It is a Gram-negative rod that can survive in myriad of environments such as aquatic and terrestrial [4]. This study was conducted to identify Pseudomonas species causing significant bacteriuria and to analyze its antibiogram in the study population.

\section{METHODS}

Institutional research committee approval was taken for the study. 107 urine samples received in the Microbiology Laboratory from August 2015 to July 2016 having significant bacteriuria with Pseudomonas species were tested for antimicrobial activity. Samples were received from both inpatients and patients attending as outpatients to a tertiary care hospital. Age and gender of the patients were noted. Midstream urine samples were inoculated using a standard calibrated loop into blood agar, MacConkey agar following semi quantitative technique using Kass concept. Streaking of the specimens was performed using four quadrant streaking method without intermittent heating on blood agar and MacConkey agar. Organism with the growth of $\geq 10^{5} \mathrm{cfu} / \mathrm{ml}$ was considered significant. MALDI (Biomerio, ElToile, France) was used for speciation, and Vitex automated system (Biomerio, ElToile, France) was used for antibacterial sensitivity testing. Antibiotics used were amikacin, ceftazidime, ciprofloxacin, gentamicin, piperacillin, aztreonam, cefoperazone-sulbactam, cefepime, imipenem, and piperacillintazobactam. Antibiotics were obtained from HiMedia, Mumbai.

\section{RESULTS}

About 107 urine samples were identified to contain Pseudomonas species by MALDI obtained from 69 (64.48\%) male patients and $38(35.51 \%)$ female patients. Inpatients were $90(84.11 \%)$ and $17(15.88 \%)$ attended to outpatient departments (OPD). Among inpatients, 64 (71\%) were males and 26 (29\%) were females and in patients attending as outpatients, 10 (59\%) were females and 7 (41\%) were males. P. aeruginosa was isolated from 90 samples (84.11\%), and others were Pseudomonas putida 17 (15.88\%). Significant bacteriuria was more in the age group of $>60$ years in both genders (Fig. 1).

Susceptibility to gentamicin was 52 (48.59\%), ceftazidime 56 (52.33\%), cefepime 58 (54.20\%), and imipenem was 58 (54.20\%). Organism was multidrug resistant in 49 (45.79\%) samples (Fig. 2).

\section{DISCUSSION}

Majority of the UTI due to Pseudomonas species was found in males when compared to females. In both the genders, UTI was more in the age group of more than 60 years. This finding varied from the studies conducted by Shah et al. [3], where females were more infected with UTI than male patients. A study conducted by Akram et al. [5] had many female patients suffering from UTI, but in the age group of 50-80 years, $100 \%$ males were suffering from UTI caused by Pseudomonas species. Schaeffer et al. [6], in their study, showed many male patients suffering from UTI than female patients, which was in concordance with our study. A study conducted by Saperston et al. [7] had more male patients presenting as inpatients with symptoms of UTI due to Pseudomonas species than outpatient presenting as UTI due to Pseudomonas species. Female patients presenting as UTI due to Pseudomonas species was 


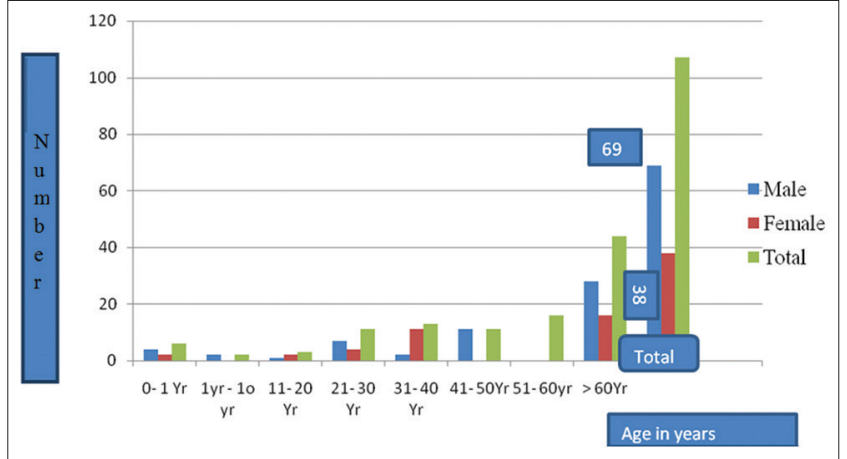

Fig. 1: Urinary tract infection in different age groups and gender

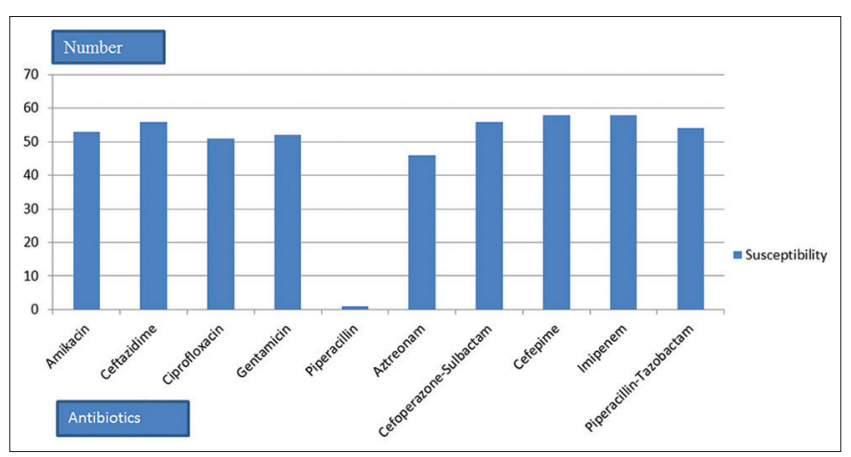

Fig. 2: Antibiotic susceptibility testing

less than male patients having UTI due to Pseudomonas species. Among the females, the number of inpatients was more than the number of outpatient suffering from UTI due to Pseudomonas species, In our study, inpatients were 90 (84.11\%) and 17 (15.88\%) attended to OPD. Out of the 90 inpatients, 64 (71\%) were males and $26(29 \%)$ were females. Male patients outnumbered the female patients among inpatients which were in concordance with the study conducted by Saperston et al. [7]. In our study, among the 17 outpatients having UTI caused by Pseudomonas species, 10 (59\%) were females and 7 (41\%) were males which did not match our study. This could be because of very small number of cases presenting as outpatients having UTI caused by Pseudomonas species in our hospital. In a study conducted by Solanki et al. [8], 31.42\% of the isolates among the nonfermenters were $P$. putida causing UTI, where as in our study only $15 \%$ of the isolates causing UTI were P. putida. P. putida was considered an opportunistic pathogen of low virulence; it can cause UTI as one of the manifestations [9]. Antibiotic pattern showed that gentamicin was susceptible in 52 (48.59\%), in our previous study [10] Pseudomonas sensitivity to gentamicin was only $37.11 \%$ [10], but the present study shows susceptibility to gentamicin was $48.59 \%$ (52 strains), this could be because of restriction of use of gentamicin after the resistance pattern, the organism could have changed its antigenicity over the period of time and now the susceptibility to gentamicin has increased. Study conducted by Biswas et al. [12] showed 40\% susceptibility to gentamicin against Pseudomonas species. This study was in concordance with our study, whereas susceptibility to amikacin was $100 \%$, but in our study, it was only $49.53 \%$. Susceptibility to ceftazidime was $52.33 \%$, where as in the study by Biswas et al. [11], had $100 \%$ susceptibility. In our previous study, susceptibility to ceftazidime was $95.45 \%$ [12], now the strains were developing resistance. Susceptibility to cefepime was 58 (54.20\%). A study conducted by Chaudhary and Payasi [11] showed a susceptibility of $42 \%$. Susceptibility to imipenem was $54.20 \%$, whereas study conducted by Biswas et al. [12] had $10 \%$ susceptibility. Our previous study had imipenem susceptibility of 95.45\% against Pseudomonas species [13]. Whereas study conducted by Syed et al. [14] showed 100\% susceptibility. A study conducted by Divyashanthi et al. [15] showed $100 \%$ susceptibility to imipenem. Moazami-Goudarzi and Eftekhar [16] in their study showed 94\% resistance to imipenem [15]. $45.79 \%$ of the organisms were multidrug resistant strains. Takeyama et al. [17] in their study showed 3 strains out of 17 Pseudomonas strains were multidrug resistant strains. This indicated that susceptibility varied from places and periodicity.

\section{CONCLUSION}

P. aeruginosa still remains the most common uropathogen among Pseudomonas species. Drug of choice for inpatients suffering from UTI by Pseudomonas species can be gentamicin and for patients attending OPD can be ceftazidime. Imipenem can be used as the reserve drug. Periodic antibiotic review is required for proper treatment of UTI.

\section{REFERENCES}

1. Tanvir R, Hafeez R, Hasnain S. Prevalence of multiple drug resistant Escherichia coli in patients of urinary tract infection registering at a diagnostic laboratory in Lahore, Pakistan. Pak J Zool 2012;44(3):707-12.

2. Wasnik DD. Prevalence and antibacterial susceptibility pattern of urinary tract infection causing human pathogenic Bacteria. Asian J Biomed Pharm Sci 2012;2(15):1-3.

3. Shah DA, Wasim S, Essa Abdullah F. Antibiotic resistance pattern of Pseudomonas aeruginosa isolated from urine samples of urinary tract infections patients in Karachi, Pakistan. Pak J Med Sci 2015;31(2):341-5.

4. Eguchi H, Miyamoto T, Kuwahara T, Mitamura S, Mitamura Y. Infectious conjunctivitis caused by Pseudomonas aeruginosa isolated from a bathroom. BMC Res Notes 2013;6:245.

5. Akram M, Shahid M, Khan AU. Etiology and antibiotic resistance patterns of community-acquired urinary tract infections in J N M C Hospital Aligarh, India. Ann Clin Microbiol Antimicrob 2007;6:4.

6. Schaeffer AJ, Nicolle LE. Clinical Practice. Urinary tract infections in older men. N Engl J Med 2016;374(6):562-71.

7. Saperston KN, Shapiro DJ, Hersh AL, Copp HL. A comparison of inpatient versus outpatient resistance patterns of pediatric urinary tract infection. J Urol 2014;191 5 Suppl:1608-13.

8. Solanki R, Dawar R, Aggarwal DK, Rani H, Imdadi F, Jasuja S. Nonfermenting gram-negative bacilli and urinary tract infectionsorting the mystery of infections caused. J Med Microbiol Diagn 4:210. Doi:10.4172/2161-0703.1000210.

9. Iwashita Y, Enokiya T, Suzuki K, Yokoyama K, Yamamoto A, Ishikura $\mathrm{K}$, et al. Arbekacin treatment of a patient infected with a Pseudomonas putida producing a metallo-beta-lactamase. J Intensive Care 2013;1:3.

10. Shobha KL, Rao GG, Kukkamalla AM. Prevalence of Non-Fermenters in Urinary Tract Infections in a Tertiary Care Hospital. Available from: http://www.webmedcentral.com. [Last accessed on 2011 Feb 07].

11. Chaudhary M, Payasi A. Comparative in vitro activity of supine against gram negative clinical isolates. Int J Pharm Pharm Sci 2015;7(7):198-202.

12. Biswas R, Rabbani R, Ahmed HS, Sarker MA, Zafrin N, Rahman MM. Antibiotic sensitivity pattern of urinary tract infection at a tertiary care hospital. Bangladesh Crit Care J 2014;2(1):21-4.

13. Shobha KL, D’Souza JS, Alva M, Anand KM, Rao G, Ramachandra L. Study of uropathogens and its antibiogram at a tertiary care hospital at coastal Karnataka. Ijppr Human 2015;4(2):100-9.

14. Syed MA, Ramakrishna PJ, Shaniya K, Arya B, Shakir VP. Urinary tract infections-an overview on the prevalence and the anti-biogram of gram negative uropathogens in a tertiary care centre in north Kerala, India. J Clin Diagn Res 2012;6(7):1192-5.

15. Divyashanthi CM, Aditiyakumar S, Bharathi N. Study of prevalence and antimicrobial susceptibility pattern of bacterial isolates in a tertiary care hospital. Int J Pharm Pharm Sci 2015;7 (1):185-90.

16. Moazami-Goudarzi1 S, Eftekhar F. Assessment of carbapenem susceptibility and multidrug-resistance in Pseudomonas aeruginosa burn isolates in Tehran. Jundishapur J Microbiol 2013;6(2):162-5.

17. Takeyama K, Kunishima Y, Matsukawa M, Takahashi S, Hirose T, Kobayashi $\mathrm{N}$, et al. Multidrug-resistant Pseudomonas aeruginosa isolated from the urine of patients with urinary tract infection. J Infect Chemother 2002;8(1):59-63. 